\title{
Protective value of dust respirators in extrinsic allergic alveolitis: clinical assessment using inhalation provocation tests
}

\author{
DJ HENDRICK, R MARSHALL, JA FAUX, JM KRALL \\ From the Department of Chest Diseases, Churchill Hospital and Radcliffe Infirmary, Oxford University, \\ Oxford and Departments of Medicine and Community Medicine, West Virginia University Medical \\ Center, Morgantown, USA
}

\begin{abstract}
Six subjects with budgerigar fancier's lung, pigeon breeder's lung, or mushroom worker's lung underwent identical paired inhalation provocation tests of 20 minutes to five hours duration (mean 2.3 hours) using a natural method of antigen exposure. For one test of each pair, the subjects were protected by the use of industrial dust respirators that incorporate electrostatically charged, resin-impregnated merino wool filters. The unprotected challenges provoked late responses of mild to moderate severity, that were characteristic of extrinsic allergic alveolitis. These were monitored by methods described previously, ${ }^{1}$ using four subjective and six objective monitoring tests. A score of 1 was allowed for each monitoring test showing a significant change. A combined monitoring score of $32 / 57$ was associated with the unprotected challenges - that is, $56 \%$ of the maximum score possible. The respirators successfully protected four of the subjects from any suspicion of a positive response. The remaining two subjects reported symptoms of minimal severity, but there was no independent confirmatory evidence of positive reactions. The combined monitoring score associated with all six protected challenges was $2 / 60(3 \%)$, while that associated with corresponding control challenges was $3 / 73(4 \%)$. We conclude that respirators of this type can offer substantial, and in most cases complete, protection against single exposures to environmental dusts that may provoke extrinsic allergic alveolitis.
\end{abstract}

A variety of respiratory protection devices are currently available for use in potentially hazardous working environments. These range from relatively simple face masks which filter out nuisance dusts of large (non-respirable) particle size, to protective suits and hoods with external air supplies that permit exposure to high concentrations of respirable agents of marked chemical, physical, or biological toxicity. Little attention has been directed towards the organic dusts responsible for extrinsic allergic alveolitis (EAA), however, and conflicting conclusions have been reached in the few reports that did consider the efficacy of respiratory protection devices for this group of disorders. The purpose of the present study was to evaluate the protection afforded by inexpensive dust respirators in subjects with EAA during exposure in natural occupational or domestic settings.

Address for reprint requests: Dr DJ Hendrick, Tulane University School of Medicine, Pulmonary Diseases Section, 1700 Perdido St, New Orleans, Louisiana 70112, USA.

\section{Methods}

Of 18 subjects reported previously ${ }^{1}$ who gave positive responses to inhalation provocation tests during the investigation of EAA, six volunteers agreed to undertake identical paired challenges using a natural method of exposure. Two were women and four were men. Their ages ranged from 28 to 54 years (mean 39 years). Two had budgerigar fancier's lung, two pigeon breeder's lung, and one mushroom worker's lung. The sixth subject had been exposed to budgerigars and canaries and was shown to be reactive to both species. Four subjects had shown the acute form of EAA and were fully recovered after a prolonged period of antigen avoidance. Two had shown the subacute form of the disease. Neither was severely affected, and both were improved after antigen avoidance.

Four other subjects served as controls with regard to the natural challenge exposures. In two, budgerigar fancier's lung and mushroom worker's lung 
respectively had been suspected, but provocation tests proved to be negative. The other two subjects (both investigators) had no evidence of active lung disease.

Informed consent was obtained from each subject and the protocol was approved by the hospital ethical committee.

\section{INHALATION TESTS}

For the natural challenge tests, four of the subjects with bird fancier's lung sat or stood in a budgerigar aviary or in one of two pigeon lofts of local breeders for periods ranging from 20 minutes to five hours. Both aviary and lofts contained about 100 healthy birds.

The budgerigar/canary fancier was a home help for a number of budgerigar-owning old age pensioners. Her paired natural challenges constituted two work periods in the same home.

The mushroom worker was also tested in his natural working environment. He returned to his usual job where he was principally engaged in distributing mushroom spawn among dried mushroom compost. The latter was moved continuously beneath him on a conveyor belt, having been loosened mechanically immediately beforehand. This weekly spawning procedure was particularly dusty. He was the employee with greatest exposure and he had related his increasingly severe acute attacks directly to it.

The duration chosen for each challenge exposure was determined largely from recent experiences. One subject with budgerigar fancier's lung was exposed for five hours. He had undergone an earlier exposure of one hour's duration which had failed to reproduce the effects noted after challenge with budgerigar serum. One pigeon fancier had become convinced that his sensitivity to pigeons had actually increased since he had given up keeping these birds himself, and a mere 20 minutes exposure was considered to be appropriate.

The paired tests were separated by intervals that ranged from six to 40 days (mean, 13 days) and were carried out under identical conditions. With three subjects, a respirator was worn on both occasions but functioning filters were fitted for only one of these. The subject was not told which. Somewhat surprisingly, characteristic odours were not readily apparent, even when the filterless respirators were worn, and the subjects were quite unable to distinguish functioning from non-functioning respirators at the end of each challenge exposure. This may have been attributable to olfactory tolerance and the unusual circumstances of exposure. With three of the paired tests, the functioning respirator was used first; and with three it was used second.
The five bird keepers also underwent control challenge tests with nebulised Coca's solution. The mushroom worker merely underwent monitoring investigations on a control day away from the mushroom farm.

The four control subjects underwent between them eight natural challenge tests involving the budgerigar aviary, a pigeon loft, or the mushroom compost.

\section{MONITORING INVESTIGATIONS}

In an earlier publication, ${ }^{1}$ we presented results of an analysis of 144 control and antigen inhalation tests carried out on 31 subjects in the investigation of bird fancier's lung, farmer's lung, and mushroom worker's lung. Changes associated with all 23 positive tests (observed in 18 reactors) were compared with changes associated with all obligatorily negative tests (control tests in the reactors, and both antigen and control tests in the 13 non-reactors). Four subjective and six objective features were found to be particularly characteristic of positive reactions. These comprised the symptoms and clinical signs of an influenza-like illness and undue respiratory effort on exercise; an increase in minute ventilation on exercise $(\geqslant+15 \%)$, body temperature $\left(>37 \cdot 2^{\circ} \mathrm{C}\right)$, circulating neutrophils $\left(\geqslant+2500 / \mathrm{mm}^{3}\right)$, and respiratory frequency on exercise $(\geqslant+25 \%)$; and a decrease in circulating lymphocytes $\left(\geqslant-500 / \mathrm{mm}^{3}\right.$ with lymphopenia), and forced vital capacity $(\geqslant-15 \%)$. The end-points associated with the objective features were chosen so that if these features were used retrospectively as confirmatory diagnostic tests, they would have specificities of about $95 \%$. The respective sensitivities ranged from $85 \%$ to $48 \%$.

By allowing a score of one for each of the four subjective and six objective monitoring tests that gave diagnostically useful changes, a maximum total monitoring score of 8-10 was possible for the overwhelming majority of inhalation tests (not every inhalation test was monitored by all 10 monitoring tests). All 23 positive inhalation tests were associated with scores of 4 or more, while all obligatorily negative tests were associated with scores of 3 or less.

The same monitoring tests were used in the present study, and monitoring scores were applied to each inhalation test in the same manner.

\section{RESPIRATORS}

Three similar types of negative pressure dust respirator were used-types $\mathrm{T}, \mathrm{W}$, and $\mathrm{X}$ from the Martindale range (Martindale Protection Ltd, Neasden Lane, London NW10 RN). All three are inexpensive costing between $£ 5$ and $£ 8$ each. Each comprises a face piece attached to an expiratory valve and an inspiratory plastic filter box (or boxes) 
containing two types of filter. The prefilter removes large, non-respirable nuisance dust thereby increasing the life of the more expensive main filter. The latter is composed of electrostatically charged, resin-impregnated merino wool, encapsulated in a plastic case. The entire cartridge is thus easily removed and replaced. B filters only were used in this study, all of which were pretested by the manufacturer (sodium chloride particulate test) to comply with the British Standard 2091, 1969.2 This requires that penetration does not exceed $2 \%$ of the respirable particles generated. The Martindale requirement was actually more stringent-a maximum penetration of $1.7 \%$.

The type $\mathrm{T}$ respirator is the least expensive. Facefit depends on standard moulding of the rubber face piece, and filtration depends on flow through one triangular B filter. Average inhaling resistance is $0.84^{\prime \prime}$ wg (water gauge) and average exhaling resistance is $0.33^{\prime \prime}$ wg.

The type $\mathrm{W}$ respirator comprises a similar facepiece but uses two triangular B filters in parallel, thereby reducing the average inhaling resistance to $0 \cdot 50^{\prime \prime}$ wg.

The type $X$ respirator (see figure) is the most expensive. It comprises a rigid plastic face-piece attached to an inflatable soft rubber cuff (for improved comfort and efficiency of face-fit), an expiratory valve and a single circular $B$ filter with greater surface area than the triangular filter. This reduces the average inhaling resistance to $0.45^{\prime \prime} \mathrm{wg}$.

\section{Results}

Data regarding the challenge exposures used, the type of respirator involved, and the results obtained are given in the table. No symptoms were experienced during the challenge periods. The six unprotected challenges provoked late reactions of mild to moderate severity. They were characterist:c of EAA, and, in the case of the bird fanciers, were essentially indistinguishable from earlier reactions to challenge with bird serum. The unprotected challenge test in

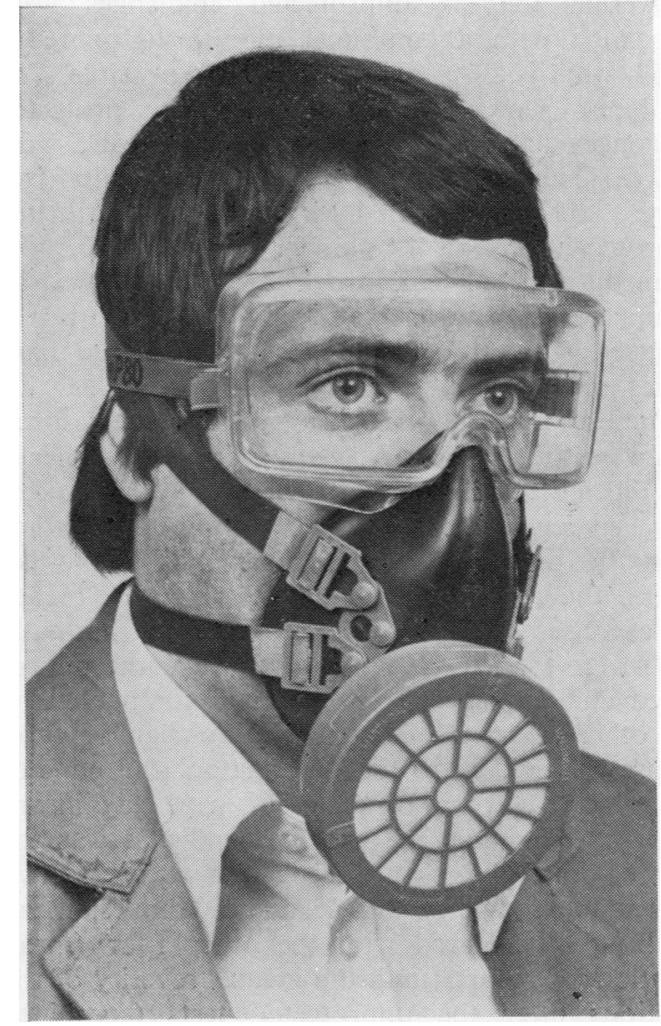

Figure Type X Martindale industrial dust respirator.

the pigeon fancier exposed for 20 minutes produced a result which was equivocal-that is, the subject concerned was convinced at the time that a definite though mild reaction had occurred, but the investigators were not fully convinced. The monitoring scores of his protected and unprotected challenges (which were indistinguishable because the respirator was worn on both occasions) proved to be 0 and 2 . This provides some confirmatory evidence that the unprotected challenge did indeed provoke a real if mild response.

Table Results of inhalation provocation tests using respiratory protection

\begin{tabular}{|c|c|c|c|c|c|c|}
\hline \multirow[t]{2}{*}{ Subject } & \multirow[t]{2}{*}{ Challenge exposure } & \multirow[t]{2}{*}{ Duration (hours) } & \multirow[t]{2}{*}{ Respirator type } & \multicolumn{3}{|c|}{ Monitoring scores } \\
\hline & & & & Unprotected & Protected & Control \\
\hline $\begin{array}{l}1 \\
2 \\
3 \\
4 \\
5 \\
6\end{array}$ & $\begin{array}{l}\text { BA } \\
\text { BA } \\
\text { BH } \\
\text { PL } \\
\text { PL } \\
\text { MF }\end{array}$ & $\begin{array}{l}5 \cdot 0 \\
1 \cdot 0 \\
4 \cdot 0 \\
1 \cdot 0 \\
0 \cdot 33 \\
2 \cdot 25\end{array}$ & $\begin{array}{l}\mathbf{X} \\
\mathbf{X} \\
\mathbf{X} \\
\mathbf{W} \\
\mathbf{T} \\
\mathbf{T}\end{array}$ & $\begin{array}{l}8 / 9 \\
5 / 10 \\
4 / 10 \\
8 / 10 \\
2 / 10 \\
5 / 8\end{array}$ & $\begin{array}{l}1 / 10 \\
0 / 10 \\
0 / 10 \\
0 / 10 \\
0 / 10 \\
1 / 10\end{array}$ & $\begin{array}{l}1 / 10 \\
0 / 10 \\
1 / 30 \\
0 / 10 \\
1 / 4 \\
0 / 9\end{array}$ \\
\hline $\begin{array}{l}\text { Total } \\
\text { Mean }\end{array}$ & & $\begin{array}{r}13 \cdot 58 \\
2 \cdot 26\end{array}$ & & $\begin{array}{l}32 / 57 \\
56 \%\end{array}$ & $\begin{array}{l}2 / 60 \\
3 \%\end{array}$ & $\begin{array}{l}3 / 73 \\
4 \%\end{array}$ \\
\hline
\end{tabular}

BA = budgerigar aviary, $\mathbf{P L}=$ pigeon loft, $\mathbf{B H}=$ budgerigar keeping home, $\mathrm{MF}=$ mushroom farm. 
Overall, the six unprotected challenges were associated with a combined monitoring score of $32 / 57$ - that is, $56 \%$ of the maximum possible. The combined scores associated with the protected challenges and corresponding control tests, were $2 / 60(3 \%)$ and $3 / 73(4 \%)$ respectively. The latter two scores, and the combined score associated with the unprotected tests in the control subjects $(2 / 77,3 \%$; mean duration of exposure 1.45 hours) showed no significant differences. The scoring system was designed so that truly negative tests should have scores not exceeding $5 \%$.

These monitoring scores suggest that the respirators were totally effective. With four of the subjects, this did indeed appear to be the case. Two subjects however reported minimal but definite symptoms after the protected challenges: one of a flu-like illness, the other of increased shortness of breath on exercise. These symptoms were substantially less severe than those experienced after the unprotected challenges. They were not accompanied by any other evidence of reactivity. No sign of a response was evident to the investigators and none of the objective monitoring tests gave significant changes. What may have been significant was the longer period of exposure associated with these two particular pairs of tests $(2.25$ and 5.0 hours, mean 3.6 hours) compared with the other four (mean duration 1.6 hours); and the greater severity of the reactions after the unprotected tests (combined monitoring scores $13 / 17,76 \%$ versus $19 / 40,48 \%$, respectively).

\section{Discussion}

There are three obvious ways of controlling environmental exposures to the organic dusts that cause EAA. The most obvious, and the best in terms of controlling the disease, is avoidance of the particular environment completely. That is, the farm worker with farmer's lung changes his job, and the pigeon fancier with pigeon breeder's lung gives up his hobby. Unfortunately, this ideal approach is not always realistic. It is not uncommon for the patient with pigeon breeder's lung to refuse to give up his birds, and farm workers often experience great difficulty when seeking other forms of employment. Furthermore, it may be the farmer himself who has the disease. Although he may reasonably delegate much of the burden of handling his hay, he will inevitably be exposed to it from time to time. In a recently published follow-up study of 92 subjects who presented with the acute form of farmer's lung, Braun and colleagues showed that $70 \%$ were still living on farms after a mean period of about 15 years. $^{3}$ Although other investigators have shown that chronic disease is not always the consequence of continued exposure, ${ }^{4}$ an appreciable risk of progressive irreversible pulmonary fibrosis does exist if exposure continues. Of the 92 subjects reported by Braun et $a l,{ }^{3}$ there was radiographic evidence of pulmonary fibrosis in $39 \%$, and diminished diffusing capacity in $30 \%$.

Two alternative methods of exposure control may be of value when the relevant environment cannot be avoided completely. Firstly, the concentration of respirable antigenic dust may be reduced to a minimum. Improved ventilation and more efficient drying of vegetable produce before storage are the most useful measures in this respect. ${ }^{56}$ Secondly, respiratory protection devices may be worn.

In their original description of pigeon breeder's lung in 1965, Reed et al reported that one of their three affected patients was able to continue handling his birds without apparent difficulty thanks to the use of a "protective mask". Crofton and Douglas, ${ }^{8}$ however, in 1969 considered the use of "masks" to be worthless in the management of farmer's lung since they permitted the passage of large numbers of small microbial spores of about $1 \mu$ in diameter. In his report to the Medical Research Council on farmer's lung in 1968, Davies ${ }^{9}$ agreed that appreciable penetration of actinomycete spores could occur through a number of respirators available commercially at the time, though he observed that dose-response relationships between spores and clinical effects had not been established. He considered that worth-while protection was likely to be provided for healthy users, but he thought that high efficiency respirators supplied with clean air under positive pressure would be necessary for farm workers who were already sensitised. Leakage from unavoidable defects in respirator-face fit would then occur only outwards. While such high efficiency devices undoubtedly provide greater protection, they are inevitably more expensive, more cumbersome, and less comfortable-especially for users engaged in sustained physical labour. Many farm workers consequently elect to work without efficient respiratory protection. ${ }^{10}$

Despite these earlier reservations, Gourley and Braidwood $^{11}$ showed in 1971 that a dust respirator using negative pressure filtration alone could fully protect a sensitised farmer from the exposure of forking a bale of his own mouldy hay for one hour. They confirmed that the filters used had efficiencies of at least $98 \%$ for $0.8 \mu$ diameter particles. Similar respirators were recommended by Siegal and Ouellette $^{12}$ to protect pigeon handlers. However, Fink et $a^{13}$ reported one case of pigeon breeder's disease, where permanent lung injury ensued despite the apparent use of a protective mask. 
The optimum particle size for alveolar deposition probably lies within the range $1-5 \mu$ in diameter. ${ }^{14}$ Smaller and larger particles may be deposited also, though in lesser proportions, and in practice virtually all alveolar deposition of organic dust is accounted for by particles $0 \cdot 2-10 \cdot 0 \mu$ in diameter. A number of comfortable and inexpensive dust respirators are now available whose filters (like those tested in the present study) are at least $98 \%$ efficient with respect to particles of this size. Efficiency measured in terms of the proportion of respirable particles penetrating (or not penetrating) through the respirator filter is not, of course, the only variable that determines the degree of clinical protection. Clinical responsiveness depends on individual reactivity and the absolute quantity of antigenic dust that is deposited within the lung. The latter can be expected to increase with increasing degrees of ambient contamination and increasing levels of ventilation (i.e. workload) irrespective of the specifications of any particular dust respirator. The salient point is that the greater the individual risk, the greater the need for a respiratory protection device. For most subjects with EAA, the repeated use of hoods or suits with independent air supplies would be impractical and only the use of negative pressure filtration respirators could be considered realistic. Their efficiency in preventing chronic disease after continued long-term exposure is quite untested, and their use in this role could only be justified if exposure levels have already been minimised, and the subject concerned is unable or unwilling to avoid the environment responsible.

The efficiency of negative pressure filtration respirators in preventing acute reactions after single exposures to organic dust is tested more readily. Our experience parallels that of Gourley and Braidwood $^{11}$ and suggests that the protection obtained can be considerable, at least in subjects engaged in sedentary or light work. Indeed, four of our six subjects appeared to have been protected completely, much to their own surprise. The two who developed symptoms after their protected challenges were quick to emphasise that these were minimal in comparison with the unprotected tests, and in these two instances no independent evidence of positive reactivity was obtained. All three types of respirator incorporated filters with identical function specifications, apart from mild differences in inspiratory resistance. They appeared to be equally efficient, though the type $\mathrm{X}$ model proved to be by far the most popular by virtue of its greater comfort.

We conclude that respirators of this type may be of considerable value in the management of acute
EAA when repeated exposure cannot be avoided. They are inexpensive and well tolerated for periods of an hour or so at a time. As a consequence, they are likely to be used if recommended.

We thank Y Hammad, DSc, for technical advice, and Martindale Protection Limited for the supply of respirators and for the illustration. The work was supported by grants from the Lauder Bequest (Oxford) and the Oxford Regional Health Authority (Teaching). The study was presented to the joint meeting of the British and Belgian Thoracic Societies in Durham in July 1980. This paper contains material from a thesis submitted by DJH to the University of London for the degree of Doctor of Medicine.

\section{References}

${ }^{1}$ Hendrick DJ, Marshall R, Faux JA, Krall JM. Positive "alveolar" responses to antigen inhalation provocation tests: their validity and recognition. Thorax 1980;35: 415-27.

${ }^{2}$ British Standards Institution. British Standard 2091, specification for respirators for protection against harmful dusts and gases. London: British Standards Institution, 1969.

${ }^{3}$ Braun SR, doPico GA, Tsiatis A, Horvath E, Dickie HA, Rankin J. Farmer's lung disease: long-term clinical and physiologic outcome. Am Rev Respir Dis 1979;119: 185-91.

${ }^{4}$ Barbee RA, Callies Q, Dickie HA, Rankin J. The longterm prognosis in farmer's lung. Am Rev Respir Dis 1968;97:223-31.

5 Grant IWB, Blyth W, Wardrop VE, Gordon RM, Pearson JCG, Mair A. Prevalence of farmer's lung in Scotland: a pilot survey. Br Med J 1972;1:530-4.

- Lehrer SB, Turer E, Weill H, Salvaggio JE. Elimination of bagassosis in Louisiana paper manufacturing plant workers. Clin Allergy 1978;8:15-20.

' Reed CE, Sosman A, Barbee RA. Pigeon-breeders' lung. A newly observed interstitial pulmonary disease. JAMA 1965;193:261-5.

${ }^{8}$ Crofton JW, Douglas AC. Respiratory diseases. Philadelphia: FA Davis Co, 1969:506-8.

9 Davies CN. Medical Research Council report-farmer's lung. Ann Occup Hyg 1968;11:377-8.

${ }^{10}$ Smyth JT, Adkins GE, Lloyd M, Moore B, McWhite E. Famer's lung in Devon. Thorax 1975;30:197-203.

${ }^{11}$ Gourley CA, Braidwood GD. The use of dust respirators in the prevention of recurrence of farmer's lung. Trans Soc Occup Med 1971 ;21:93-5.

${ }^{12}$ Siegal FP, Ouellette JJ. Protecting pigeon-handlers. Lancet $1969 ; 1: 733-4$.

${ }^{13}$ Fink JM, Sosman AJ, Barboriak JJ, Schlueter DP, Holmes RA. Pigeon breeder's disease-a clinical study of a hypersensitivity pneumonitis. Ann Intern Med 1968; 68:1205-19.

${ }^{14}$ Muir DCF. Deposition and clearance of inhaled particles. In: Muir DCF, ed. Clinical aspects of inhaled particles. Philadelphia: FA Davis Co, 1972:7-8. 